\title{
Subjektive Gedächtnisbeeinträchtigungen in der Früherkennung von Demenzen: Beziehung zur Akzeptanz der Lumbalpunktion bei Patienten einer Gedächtnissprechstunde
}

\section{Subjective memory impairments in early detection of dementia: Relation to acceptability of lumbar puncture in memory clinic patients}

Autoren

Nicole Cabanel ${ }^{1}$, Christa Speier ${ }^{2}$, Michael Franz ${ }^{3}$, Matthias J. Müller ${ }^{4}$, Bernd Kundermann ${ }^{1}$

Institute

1 Vitos Klinikum Gießen-Marburg, Vitos Klinik für Psychiatrie und Psychotherapie Gießen; Fachbereich Medizin, Philipps-Universität Marburg

2 Vitos Klinikum Gießen-Marburg, Vitos Klinik für Psychiatrie und Psychotherapie Gießen

3 Vitos Klinikum Gießen-Marburg, Vitos Klinik für Psychiatrie und Psychotherapie Gießen; Fachbereich Medizin, Justus-Liebig-Universität Gießen

4 Oberberggruppe, Berlin; Fachbereich Medizin, JustusLiebig-Universität Gießen

Schlüsselwörter

Gedächtnissprechstunde, Subjektive

Gedächtnisbeeinträchtigungen, Demenzdiagnostik,

Lumbalpunktion

Key words

memory clinic, subjective memory impairment, dementia

diagnostics, lumbar puncture

eingereicht 24.01 .2018

akzeptiert $\quad 14.06 .2018$

Bibliografie

DOI https://doi.org/10.1055/a-0644-2530

Fortschr Neurol Psychiatr 2018; 86: 298-304

(c) Georg Thieme Verlag KG Stuttgart · New York

ISSN 0720-4299

Korrespondenzadresse

Dr. phil. Dipl.-Psych. Bernd Kundermann

Vitos Klinikum Gießen-Marburg, Vitos Klinik für Psychiatrie und Psychotherapie Gießen

Licher Str. 106

35394 Gießen

bernd.kundermann@vitos-giessen-marburg.de

\section{ZUSAMMENFASSUNG}

Hintergrund In Gedächtnissprechstunden kommt der biomarkergestützten Diagnostik eine zunehmend wichtige Rolle in Früherkennung und Differentialdiagnose von Demenzen zu, wenngleich deren Akzeptanz bei Patienten vergleichsweise niedrig ist.

Ziel der Arbeit Es wurde untersucht, ob soziodemographische und klinische Variablen von Patienten einer Gedächtnissprechstunde mit der Adhärenz zur Lumbalpunktion (LP) assoziiert sind. Von besonderem Interesse war die vom Patienten selbst wahrgenommene Verschlechterung des Gedächtnisses (subjektive Gedächtnisbeeinträchtigung, SGB) mit hierauf gerichteter Besorgnis, die einen Einfluss auf die Entscheidung für eine LP ausüben könnte.

Methode Konsekutive Patienten wurden einer tagesklinischen Abklärung an zwei aufeinanderfolgenden Tagen zugeführt, um ein an der S3-Leitlinie „Demenzen“ angelehntes diagnostisches Vorgehen inkl. Angebot einer LP umzusetzen. Es wurde Depressivität, Angst, neurokognitive Leistung und der Demenzschweregrad (Clinical Dementia Rating, CDR) erhoben. Ebenso wurden die Patienten zu selbst wahrgenommenen Gedächtnisverschlechterungen interviewt und auf dieser Grundlage - unabhängig ihrer neuropsychologischen Befunde - drei Gruppen zugeordnet: keine SGB, SGB ohne Besorgnis oder SGB mit Besorgnis.

Ergebnisse Von 44 Patienten (73,8 $\pm 8,3$ Jahre; $27 \mathrm{w} / 17 \mathrm{~m}$; CDR<1: $n=16, C D R=1: n=28$ ) zeigten 29 SGB mit Besorgnis. Sie waren tendenziell jünger und hatten einen höheren Bildungsstatus als Patienten ohne SGB $(n=7)$ und Patienten mit SGB ohne Besorgnis ( $n=8)$. Patienten ohne SGB hatten häufiger ein Demenzsyndrom. Zur LP einwilligende Patienten $(n=23)$ waren - gegenüber 17 ablehnenden Patienten (4 Patienten wurden aufgrund medizinischer Kontraindikation für unmittelbare LP ausgeschlossen) - tendenziell häufiger männlichen Geschlechts, zeigten signifikant häufiger SGB mit Besorgnis und geringere Leistungen in deklarativen Gedächtnisaufgaben. In einer binärlogistischen Regressionsanalyse verblieben männliches Geschlecht und SGB mit Besorgnis wie auch verminderte Gedächtnisleistungen als bedeutsame Prädiktoren für die Einwilligung zu einer LP. 
Schlussfolgerung Die Studie deutet darauf hin, dass Patientencharakteristika einer subjektiven - mit Besorgnis einhergehenden - und objektiven Gedächtnisbeeinträchtigung sowie auch das Geschlecht die Einwilligung zu einer invasiven und im Allgemeinen weniger akzeptierten biomarkergestützten Diagnostik wie der LP mitbedingen.

\section{ABSTRACT}

Background In memory clinics, biomarker-based diagnostic tools for early detection and differential diagnosis of dementia are increasingly important, even if their acceptance by patients is relatively low.

Objective The aim of study was to examine whether sociodemographic and clinical features of memory clinic patients are associated with acceptance of lumbar puncture (LP). Of particular interest was the patients' self-perception of memory decline (subjective memory impairment, SMI) accompanied by related concerns that might affect decision to consent to LP.

Methods Consecutive patients were examined in a day-care hospital on two consecutive days in order to implement a diagnostic procedure based on the S3 guideline "Dementia" including offer of LP. We assessed demographic and clinical variables such as depression, anxiety, neurocognitive performance and dementia severity (Clinical Dementia Rating, CDR). Furthermore, patients were interviewed about perceived memory decline and were classified on this basis independent of their neuropsychological results - into three groups: no SMI, SMI without concerns or SMI with concerns. Results Of 44 patients $(73.8 \pm 8.3$ years; $27 \mathrm{f} / 17 \mathrm{~m}$; CDR < 1: $n=16, C D R=1: n=28), 29$ had SMI with concerns. These patients tended to be younger and had a higher level of education than those who did not report SMI $(n=7)$ and those perceiving SMI without concerns $(n=8)$. Furthermore, patients without SMI more frequently had a dementia syndrome. Patients who agreed to lumbar puncture $(n=23)$ were - compared to patients refusing LP $(n=17 ; 4$ patients had to be excluded because of medical contraindication for immediate LP) - more likely male, had significantly more frequent SMI with concerns and performed poorer on declarative memory tasks. Binary regression analysis yielded SMI with concerns, a more impaired memory performance and male sex as significant predictors for consenting to LP.

Conclusions The study provides evidence that patient characteristics such as subjective and objective memory impairment as well as sex may affect the likelihood to consent to a generally less accepted biomarker-based dementia diagnostic procedure such as LP.

\section{Einleitung}

In Gedächtnissprechstunden wird in der Regel ein sehr heterogenes Klientel vorstellig, das Patienten mit neurodegenerativen Erkrankungen, anderen Hirnfunktionsstörungen, psychischen Störungen sowie „gesunde Besorgte“ umfasst [1]. Zur Früh- und Differentialdiagnose der Alzheimer-Demenz gewinnen biomarkergestützte Methoden wie die liquorbasierte Demenzdiagnostik mittels Lumbalpunktion (LP) eine zunehmend größere Bedeutung [2]. Der fortschreitenden Entwicklung dieser biomarkergestützten Diagnostik steht allerdings gegenüber, dass deren Anwendung - im Vergleich zur Durchführung nicht-invasiver und vertrauterer Methoden - in Patientenbefragungen weniger akzeptiert ist [3], was sich auch in Durchführungs- bzw. Ablehnungshäufigkeiten [4] ausdrückt. Dies kann mit untersuchungsimmanenten Nebenwirkungen und Risiken der LP zusammenhängen, aber auch die Bedeutung der individuellen Abwägung der Folgen einer Frühdiagnostik [5] wird in diesem Zusammenhang diskutiert.

Bislang wurde nicht untersucht, ob Patientenmerkmale mit der Akzeptanz (i. S. einer konkreten Einwilligung und Durchführung) einer invasiven biomarkergestützten Diagnostik assoziiert sind. Hinweise auf mögliche Zusammenhänge finden sich in einer früheren Arbeit von Boustani et al [6], aus der eine höhere Ablehnungsquote gegenüber einer erweiterten Demenzdiagnostik bei Patienten mit besseren kognitiven Leistungen im Screening hervorging. Ebenso scheint bei Patienten einer Gedächtnissprechstunde das subjektive Erleben kognitiver Insuffizienz - stärker als objektivierbare kognitive Leistungsminderungen - in den Entscheidungsprozess zugunsten einer diagnostischen Abklärung involviert zu sein [7]. Einen theoretischen Rahmen dieser Befunde bietet u. a. das Common Sense Model of Illness Representation [8], das die individuelle Auseinandersetzung und Bewertung von Symptomen in Bezug zur Inanspruchnahme von medizinischer Hilfe sieht.

Das subjektive Erleben kognitiver Störungen ist Gegenstand eines zunehmenden Forschungsinteresses an ersten symptomatischen Anzeichen des präklinischen Stadiums der AlzheimerErkrankung. Die subjektive kognitive Verschlechterung (subjective cognitive decline, SCD), die entsprechend den Forschungskriterien als vom Patienten erlebte Einbuße in mnestischen und/ oder nicht-mnestischen Funktionen bei normgerechter Leistung in neuropsychologischen Tests definiert ist (weitere Voraussetzungen sind u.a., dass diese nicht durch eine andere psychische Störung erklärbar ist), erwies sich in longitudinalen Studien als Risikofaktor für einen fortschreitenden kognitiven Abbau entlang des Kontinuums zur leichten kognitiven Beeinträchtigung (mild cognitive impairment, $\mathrm{MCl}$ ) bis hin zur Alzheimer-Demenz [9]. Andere Konzepte fokussierten hingegen spezifische Einbußen im Bereich des Gedächtnisses (subjektive Gedächtnisbeeinträchtigungen, SGB) und differenzierten diese weiter nach hierauf bezogener Besorgnis („memory concerns“) [10]. Die Definition von SGB setzte in einigen Studien jedoch - im Unterschied zu den SCD-Forschungskriterien [9] - keinen normgerechten neuropsychologischen Status voraus, so dass auch Patienten mit $\mathrm{MCl}$ und Demenzsyndrom anhand ihrer SGB charakterisiert wurden [11]. Wie aus der Studie von Jessen et al. [12] hervorgeht, waren SGB 
mit Besorgnis - gegenüber SGB ohne Besorgnis - mit einem signifikant höheren Risiko für eine Konversion zu einer Demenz assoziiert, womit zumindest der prädiktive Wert einer differenzierten Erfassung von SGB für den weiteren Verlauf unterstrichen wurde.

Das Ziel der Studie ist es, Patienten einer Gedächtnissprechstunde im Hinblick auf SGB und hiermit assoziierte Variablen zu beschreiben und zu explorieren, ob SGB sowie weitere soziodemographische und klinische Merkmale mit der Adhärenz zu einer invasiven und biomarkergestützten diagnostischen Maßnahme (LP) zusammenhängen. Aufgrund der berichteten Befunde und theoretischer Erwägungen zur Bedeutung von subjektiven Faktoren (v.a. Wahrnehmung und Bewertung von Symptomen) für die Inanspruchnahme medizinischer Leistungen wird erwartet, dass SGB in Verbindung mit hierauf bezogener Besorgnis die Entscheidungsbildung zugunsten einer Zustimmung zur LP begünstigt.

\section{Methodik}

\section{Untersuchungsablauf und Patienten}

An einer Gedächtnissprechstunde eines Fachklinikums für Psychiatrie und Psychotherapie wurden zwischen November 2015 und August 2017 Patienten für eine Längsschnittstudie („Früherkennung und Differenzialdiagnose kognitiver Störungen in einer Gedächtnisambulanz") rekrutiert. Die Patienten durchliefen ein strukturiertes diagnostisches Vorgehen im ambulanten und teilstationären Rahmen an zwei aufeinanderfolgenden Tagen entsprechend der S3-Leitlinie Demenzen [13], ebenso wurden weitere Untersuchungen berücksichtigt ( $\mathbf{A}$ Abb. 1 $)^{1}$. Die Studienteilnahme setzte eine fachärztliche Indikation für eine erweiterte Demenzabklärung, Einwilligungsfähigkeit und ein Mindestalter von 51 Jahren voraus. Als Ausschlusskriterium galt eine mehr als zwei Jahre bestehende Demenzdiagnose. Alle Patienten gaben vor Studienbeginn ihre schriftliche Einwilligung. Die Studie wurde mit Zustimmung der Ethikkommission der Landesärztekammer Hessen durchgeführt und ist im Deutschen Register Klinischer Studien unter DRKS00010215 registriert.

\section{Variablen und deren Erfassung}

Subjektive Gedächtnisbeeinträchtigungen (SGB): Die Patienten wurden - in Anlehnung an Geerlings et al [10] - zu zwei Fragen interviewt: „Haben Sie das Gefühl, dass Ihr Gedächtnis schlechter geworden ist?“ (ja / nein), sowie „Falls ja, macht es Ihnen Sorgen?“ (ja/nein). Auf dieser Grundlage wurden die Patienten „- unabhängig ihres neuropsychologischen und diagnostischen (ICD-10) Status -“ drei Gruppen zugeordnet: keine SGB, SGB ohne Besorgnis und SGB mit Besorgnis.

Kognitiver Leistungsstatus und Syndromschwere: Aus der neuropsychologischen Testbatterie CERAD-plus [14] wurden

${ }^{1}$ Für die vorliegende Arbeit wurde auf die Auflistung weiterer Untersuchungsvariablen und -messungen (u. a. circadiane Präferenz, biologische Stressreaktivitätsmarker, Aktigraphie etc.) der longitudinal-konzipierten Gesamtstudie verzichtet.

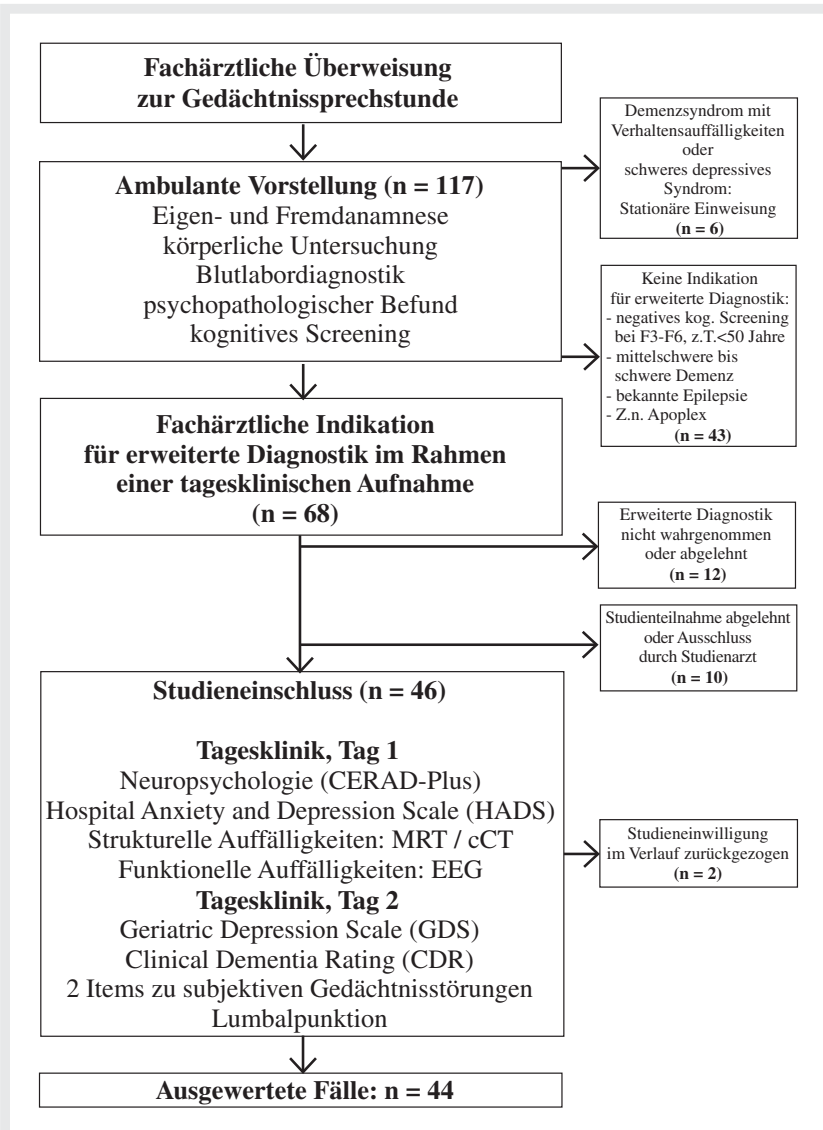

- Abb. 1 Untersuchungsablauf im ambulanten und tagesklinischen Rahmen

zwei globale Leistungsscores generiert: der Rohwert des MiniMental-Status-Test (MMST) und ein aus der CERAD-Plus extrahierter Memory Total Score (CMTS) [15]. Die Clinical Dementia Rating Scale (CDR) [16] diente der Einschätzung des Demenzschweregrades.

Angst und Depressivität: Hierzu wurde die deutsche Version der Hospital Anxiety and Depression Scale (HADS) [17] eingesetzt, ergänzend wurde die Kurzform (15 Items) der Geriatrischen Depressionsskala (GDS) [18] als Interview durchgeführt.

Weitere Untersuchungen und Variablen: Die Studienärzte waren instruiert, jedem Patienten eine LP anzubieten und diese-unter Beachtung der Patientensouveränität-mit dem Empfehlungsgrad der S3-Leitlinie (Expertenkonsens, „Kann“Empfehlung) zu begründen. Die diesbezügliche standardisierte Aufklärung erfolgte an Tag 1, die Einholung der Einwilligung und Durchführung an Tag 2. Ebenso wurde eine Elektroenzephalographie und Magnetresonanz- oder kraniale Computertomographie angeboten, die im Hinblick auf Durchführung vs. Ablehnung registriert wurden. Die Patienten wurden syndromal klassifiziert in a) Subjektive kognitive Verschlechterung (subjective cognitive decline. ohne neuropsychologisch nachweisbaren kognitiven Defizite entsprechend den Forschungskriterien [9], b) $\mathrm{MCl}$ entweder ohne oder in Verbindung mit 
einer anderen psychischen Störung oder c) Demenz nach den allgemeinen ICD-10 Syndromkriterien. Die Spezifizierung der Diagnose erfolgte nach ICD-10.

Statistische Auswertung: Die Datenanalyse erfolgte mit dem Statistikprogramm R. Neben deskriptiven Statistiken (Häufigkeiten, Mittelwerte \pm Standardabweichung) und Gruppenvergleichen (Fishers exakter Test, Mann-Whitney U-Test) wurde eine binäre logistische Regression zur Vorhersage der Durchführung einer LP durch klinische und soziodemographische Merkmale vorgenommen. Das Signifikanzniveau wurde auf $\mathrm{P}<0,05$ festgelegt.

\section{Ergebnisse}

\section{Stichprobenbeschreibung}

Der Tab. 1 sind die soziodemographischen und klinischen Charakteristika der Stichprobe zu entnehmen.

- Tab. 1 Demographische und klinische Charakteristika der Patienten

\begin{tabular}{|c|c|}
\hline Variable & $M \pm S D$ bzw. $n$ \\
\hline Alter [Jahre] & $73,8 \pm 8,3$ \\
\hline Geschlecht [w/m] & $27 / 17$ \\
\hline Bildung [Jahre] & $11,4 \pm 3,0$ \\
\hline $\begin{array}{l}\text { Diagnose nach ICD-10 } \\
\text { - } \quad \text { Alzheimer Demenz [F00.0 / F00.1/ F00.2] } \\
\text { - } \text { Vaskuläre Demenz [F01.2 / F01.3] } \\
\text { - } \quad \text { Nicht näher bezeichnete Demenz [F03] } \\
\text { rungen aufgrund einer Krankheit, Schädigung } \\
\text { oder Funktionsstörung des Gehirns [F07.8] } \\
\text { - } \quad \text { Depressive Episode [F32.x/ F33.x] } \\
\text { - } \quad \text { Keine ICD-10 F-Diagnose [Z03.x] }\end{array}$ & $\begin{array}{l}4 / 9 / 8 \\
4 / 1 \\
2\end{array}$ \\
\hline $\begin{array}{l}\text { Kognitive Syndromdiagnose }\left[\mathrm{SCD}^{3} / \mathrm{MCl} /\right. \\
\text { Demenz] }\end{array}$ & $1 / 15 / 28$ \\
\hline $\begin{array}{l}\text { Psychopathometrie: } \\
\text { - Clinical Dementia Rating (CDR) }[0 / 0,5 / 1]\end{array}$ & $3 / 13 / 28$ \\
\hline - Mini-Mental Status Examination (MMSE) & $24,0 \pm 3,4$ \\
\hline - CERAD Memory Total Score (CMTS) & $25,0 \pm 5,5$ \\
\hline - Geriatric Depression Scale (GDS) & $6,2 \pm 3,2$ \\
\hline $\begin{array}{l}\text { - Hospital Anxiety and Depression Scale (HADS) } \\
\text { - Angst } \\
\text { - Depression }\end{array}$ & $\begin{array}{l}5,5 \pm 4,5 \\
6,0 \pm 4,9\end{array}$ \\
\hline
\end{tabular}

${ }^{2}$ Ätiologische Differenzierung: Hinweise auf neurodegenerative $(n=6)$, vaskuläre $(n=2)$ und gemischte $(n=2)$ Genese.

${ }^{3}$ Subjective cognitive decline (subjektive kognitive Verschlechterung) ohne neuropsychologisch nachzuweisende Defizite und nicht durch eine andere psychische Störung erklärbar [9].
Gruppenvergleiche zwischen Patienten anhand ihrer subjektiven Gedächtnisbeeinträchtigungen (SGB)

Aus Abb. 2 geht die Klassifikation der Stichprobe anhand ihrer SGB hervor. Die Majorität der Patienten war durch SGB mit Besorgnis $(n=29)$ charakterisiert, während $n=7$ keine SGB und $n=8$ SGB ohne Besorgnis zeigten. Die nach Kategorien zusammengefassten ICD-10 Diagnosen verteilten sich über diese Gruppen wie folgt: SGB mit Besorgnis: F00.x $(n=13)$, F01.x $(n=3)$, F03 $(n=2)$, F07.x $(n=7)$, F3 $(n=3)$ und Z03.x $(n=1)$; SGB ohne Besorgnis: F00.x $(n=2)$, F01.x $(n=1)$, F07.x $(n=3)$ und F3 $(n=2)$; keine SGB: F00.x $(n=6)$ und F01.x $(n=1)$. Hieraus geht auch hervor, dass der Anteil von Patienten mit Demenzsyndrom $(C D R \geq 1)$ gegenüber solchen mit $C D R<1$ ( $S C D$ und $M C l$ ) über die drei Gruppen ungleich verteilt war ( $\mathbf{A} \mathbf{A b} \mathbf{b} . \mathbf{2})$ : ein Demenzsyndrom lag bei Patienten ohne SGB $(n=7,100 \%)$ im Vergleich zur Gruppe SGB ohne Besorgnis $(n=3,37,5 \%)$ und SGB mit Besorgnis ( $n=18,62 \%$ ) überproportional häufig vor $(P=0,033)$. Darüber hinaus zeigte sich, dass Patienten mit SGB und Besorgnis (71,6 $\pm 9,2$ Jahre) tendenziell jünger waren als Patienten ohne SGB $(78,6 \pm 4,5$ Jahre) und Patienten mit SGB ohne Besorgnis $(77,8 \pm 3,2$ Jahre) ( $P$ jeweils $<0,1)$. Die Gruppe SGB mit Besorgnis hatte gegenüber Patienten mit SGB ohne Besorgnis mehr Bildungsjahre (12,2 $\pm 2,8$ Bildungsjahre vs. 9,4 $\pm 3,1$; $P=0,03)$, während der Unterschied zu Patienten ohne SGB $(10,0 \pm 2,1)$ nur tendenziell $(P=0,059)$ ausfiel. Aus den neuropsychologischen Maßen der Gruppen SGB mit Besorgnis (MMST: 24,1 $\pm 3,6$; CMTS: 24,8 $\pm 5,6$ ), SGB ohne Besorgnis $(24,0 \pm 3,5$; CMTS: $26,4 \pm 5,1)$ und keine SGB (MMST: 23,3 $\pm 2,2$; CMTS: $24,7 \pm 6,4$ ) gingen keine bedeutsamen Differenzen hervor, ebenso bestanden keine signifikanten Gruppenunterschiede in anderen klinischen Variablen (Angst, Depressivität).

\section{Assoziationen zwischen soziodemographischen und klinischen Charakteristika zur Adhärenz diagnostischer Maßnahmen}

Die Adhärenzquote zu allen in \$ Abb. 1 genannten diagnostischen Maßnahmen lag - mit Ausnahme der LP - bei jeweils $100 \%$. Bei 4 Patienten mit oraler Antikoagulation konnte keine LP im Kontext der zweitägigen teilstationären Abklä-

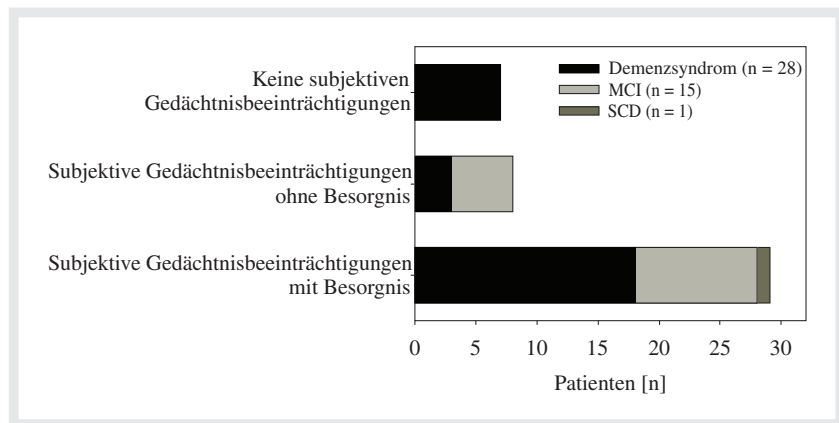

-Abb. 2 Häufigkeitsverteilung der Patienten im Hinblick auf subjektive Gedächtnisbeeinträchtigungen unter Berücksichtigung der kognitiven Syndromdiagnose (Demenzsyndrom, MCl und SCD) 
Tab. 2. Gruppenvergleich zwischen Patienten, die eine Lumbalpunktion (LP) ablehnten vs. lumbalpunktierten Patienten ${ }^{4}$

\begin{tabular}{|c|c|c|c|}
\hline \multirow[t]{2}{*}{ Variable } & \multicolumn{2}{|c|}{ LP } & \multirow{2}{*}{$\begin{array}{c}\text { Inferenzstatistik } \\
\text { P-Werte (zweiseitig) }\end{array}$} \\
\hline & $\begin{array}{l}\text { Abgelehnt ( } n=17) \\
M \pm S D \text { bzw. } n\end{array}$ & $\begin{array}{l}\text { Durchgeführt }(n=23) \\
\qquad M \pm S D \text { bzw. } n\end{array}$ & \\
\hline Alter [Jahre] & $79,9 \pm 8,4$ & $72,3 \pm 8,8$ & 0,212 \\
\hline Geschlecht [w/m] & $14 / 3$ & $12 / 11$ & 0,092 \\
\hline Bildung [Jahre] & $10,9 \pm 3,3$ & $11,4 \pm 2,6$ & 0,813 \\
\hline Clinical Dementia Rating (CDR) $[<1 / \geq 1]$ & $8 / 9$ & $6 / 17$ & 0,198 \\
\hline Mini-Mental Status Examination (MMSE) & $24,9 \pm 3,5$ & $23,1 \pm 3,3$ & 0,127 \\
\hline CERAD Memory Total Score (CMTS) & $26,6 \pm 6,2$ & $23,7 \pm 4,0$ & 0,040 \\
\hline Geriatric Depression Scale (GDS) & $6,3 \pm 4,0$ & $5,8 \pm 2,8$ & 0,762 \\
\hline $\begin{array}{l}\text { Hospital Anxiety and Depression Scale (HADS) } \\
\text { - Angst }\end{array}$ & $4,8 \pm 5,6$ & $5,8 \pm 3,8$ & 0,169 \\
\hline - Depression & $5,3 \pm 6,0$ & $6,5 \pm 4,6$ & 0,206 \\
\hline $\begin{array}{l}\text { Subjektive Gedächtnisbeeinträchtigungen (SGB) } \\
\text { - (1) Keine SGB } \\
\text { - (2) SGB ohne Besorgnis } \\
\text { - (3) SGB mit Besorgnis }\end{array}$ & $\begin{array}{l}4 \\
5 \\
8\end{array}$ & $\begin{array}{c}2 \\
3 \\
18\end{array}$ & 0,161 \\
\hline
\end{tabular}

rung durchgeführt werden. Von 40 verbliebenen Patienten lehnten 17 Patienten (42,5\%) die LP explizit ab, während diese bei 23 Patienten (57,5\%) nach Einwilligung durchgeführt wurde.

Aus Tab. 2 geht hervor, dass zur LP einwilligende Patienten tendenziell häufiger männlichen Geschlechts waren und einen signifikant niedrigeren Gedächtnisscore (CMTS) als Patienten der LP-ablehnenden Gruppe aufwiesen $(P<0,05)$. SGB erwiesen sich deskriptiv v.a. dann mit der Einwilligung und Durchführung einer LP assoziiert, wenn sie mit Besorgnis einhergingen. Dennoch erwies sich diese unterschiedliche Häufigkeitsverteilung als statistisch nicht signifikant. Bei Zusammenfassung der beiden ersten Gruppen zu $n=14$ (jeweils Patienten ohne Besorgnis) ergab sich im Vergleich zur Gruppe SGB mit Besorgnis $(n=26)$ bei hypothesengeleiteter einseitiger Prüfung ein signifikanter Unterschied $(P=0,044)$, der die höhere Rate von SGB mit Besorgnis bei LP-einwilligenden Patienten ausdrückte. Ebenso wurde eine binär logistische Regressionsanalyse mit der Kriteriumsvariable „LP durchgeführt“ (nein vs. ja) vorgenommen. Geschlecht, CERAD Memory Total Score (CMTS) sowie SGB als dichotome Variable (mit Besorgnis: nein vs. ja) wurden als Prädiktoren berücksichtigt. Das Regressionsmodell erwies sich als signifikant (Nagelkerke $R^{2}=0,405 ;$ Modell $X_{(3)}^{2}=14,354 ; P=0,002$ ), wobei Geschlecht (Wald $X^{2}(1)=5,525 ; P=0,019$ ), der CMTS

${ }^{4}$ Patienten mit oraler Antikoagulation wurden von dieser Analyse ausgeschlossen
(Wald $X_{(1)}^{2}=4,373 ; P=0,037$ ) und SGB mit Besorgnis (Wald $\left.X^{2}(1)=4,008 ; P=0,045\right)$ einen statistisch bedeutsamen Beitrag zu einer korrekten Zuordnung von 75\% der Fälle leisteten.

\section{Diskussion}

Die vorliegende Arbeit untersuchte, ob bei konsekutiv aufgenommenen Patienten einer Gedächtnissprechstunde die Durchführung einer Lumbalpunktion (LP) im Rahmen einer erweiterten Demenzdiagnostik mit soziodemographischen oder klinischen Merkmalen assoziiert ist. Im Fokus der Betrachtung lag die Bedeutung von subjektiven Gedächtnisbeeinträchtigungen (SGB).

Im Vergleich zu anderen vorgenommenen diagnostischen Maßnahmen (Neuropsychologie, EEG, MRT) ging eine geringere Adhärenz für die LP hervor, womit vorliegende Befunde unterstützt wurden [3]. Die beobachtete LP-Anwendungsquote (57,5\%) lag auch unterhalb derer aus anderen Arbeiten, an größtenteils universitären Gedächtnissprechstunden [z. B. 19], so dass diese Diskrepanz auch mit settingbezogenen Unterschieden (mit vergleichsweise hoher Bereitschaft zur LP bei Patienten einer universitären Gedächtnissprechstunde [20]) erklärt werden könnte. Bei aufsuchenden Patienten einer Gedächtnisambulanz eines psychiatrisch-psychotherapeutischen Versorgungsklinikums könnte diese Bereitschaft geringer ausgeprägt sein, wenngleich die Repräsentativität bei unserer Stichprobe gegenüber Studien mit Patienten aus rein universitären Einrichtungen eher gegeben sein dürfte (ökologische Validität). 
Die Analyse patientenbezogener Faktoren bestätigte unsere Hypothese, dass SGB mit Besorgnis mit einer größeren Akzeptanz zur Durchführung einer LP einherging. LP-einwilligende zeigten gegenüber LP-ablehnenden Patienten jedoch auch stärkere objektive Gedächtnisdefizite, während andere Symptomdimensionen (Angst, Depression) als auch soziodemographische Merkmale nicht differierten. Letzterer (negativer) Befund ist unter Vorbehalt zu interpretieren, da die geringe statistische Power der Studie einen Typ-II-Fehler begünstigt. Andererseits war diese zur Abbildung von Gruppenunterschieden zumindest in objektiven kognitiven Dysfunktionen ausreichend, so dass der kognitiven Symptomatik möglicherweise eine spezifischere Bedeutung für die Adhärenz zugunsten einer LP zukommt.

Eine Einordnung dieser Befunde im Zusammenhang anderer Studienergebnisse ist dadurch limitiert, dass die Beziehung von SGB zur LP-Adhärenz bislang nicht untersucht wurde. Eine aktuelle Arbeit [21] berichtet, dass Besorgnis vor einer Alzheimer-Demenz mit einer erhöhten Akzeptanz zur Diagnostik einherging, auch für somatische Erkrankungen konnten entsprechende Zusammenhänge gezeigt werden [22]. Die Befunde könnten dafür sprechen, dass eigens wahrgenommene und als gesundheitsbedrohlich bewertete kognitive Symptome die Entscheidungsbildung zugunsten der Inanspruchnahme weniger akzeptierter medizinischer Maßnahmen begünstigen [8]. Kritisch ist anzumerken, dass SGB in Studien sehr heterogen definiert und erfasst werden [23]. Auch die Operationalisierung von Geerlings et al [10] ist unscharf, da nicht weiter differenziert wird, worauf sich die Sorgen angesichts wahrgenommener SGB richten. Diese könnten subjektiv erlebte Auswirkungen im Alltag (d. h. funktionelle Aspekte) betreffen oder sich auf eine zugrundliegende (Alzheimer-) Pathologie im Sinne von „dementia worries“ [24] beziehen, wobei für letzteres Überlappungen zur Krankheitsangst („neurocognitive hypochondriasis“ [25]) bestehen.

Es fiel auf, dass bei allen Patienten ohne SGB (16\%) eine Demenz vorlag. Diese Subgruppe könnte Patienten mit einer Anosognosie für kognitive Defizite repräsentieren, wenngleich andere Studien - bei jedoch sensitiverer Erfassung (z. B. mittels Diskrepanzscores zwischen Selbsteinschätzung und neuropsychologischen Maßen) - höhere Prävalenzraten (z. B. 42 \% bei leichter Alzheimer-Demenz) berichten [26]. Bei den MCI-Patienten zeigte sich eine relative Häufigkeit von SGB mit Besorgnis von $67 \%$. Dieser Anteil fiel deutlich größer aus als bei Jessen et al [27] (33\% der MCl-Patienten), die jedoch ausschließlich Patienten aus der hausärztlichen Versorgung inkludierten.

Als Nebenbefund ging hervor, dass in unserer Stichprobe männliches Geschlecht mit einer erhöhten Bereitschaft zur Durchführung einer LP einherging. Geschlechtseffekte gingen bislang ausschließlich aus Studien hervor, die allgemeine Einstellungen zur Frühdiagnostik untersuchten. Die Datenlage hierzu ist sehr heterogen mit vergleichbaren [28] wie auch diametralen [21] Befunden. Ebenso ist kritisch anzumerken, dass eine statistische Kontrolle von Kovariaten oder eine Analyse von Subgruppen (z.B. männlich und SGB mit Besorgnis) aufgrund der Stichprobengröße nicht vorgenommen werden konnte.
Abschließend ist der diagnostische Wert der LP im Rahmen einer Gedächtnissprechstunde hervorzuheben, da-wie auch die Diagnoseverteilung unserer Studienpatienten zeigte-Patienten mit kognitiven Störungen vor dem Hintergrund gut behandelbarer (z. B. depressiver) Erkrankungen hiermit zuverlässiger identifiziert werden können [2]. Hingegeben bedürfen SCD- und $\mathrm{MCl}$ Patienten mit positivem Liquorbefund einer sensiblen Aufklärung und Beratung, die nicht nur auf den erhöhten Risikostatus für einen (weiteren) kognitiven Abbau ausgerichtet ist, sondern auch die Relevanz prospektiver Verlaufsdiagnostik hervorhebt und über verlaufs- bzw. stadienabhängige psychosoziale wie psychopharmakologische Therapieoptionen informiert.

\section{FAZIT FÜR DIE PRAXIS}

Aus der Studie geht hervor, dass Patientencharakteristika einer subjektiven - mit Besorgnis einhergehenden - und objektiven Gedächtnisbeeinträchtigung sowie auch das Geschlecht die Einwilligung zu einer invasiven und im Allgemeinen weniger akzeptierten biomarkergestützten Diagnostik wie der Lumbalpunktion mitbedingen. Darüber hinaus wurde gezeigt, dass im Rahmen von Gedächtnissprechstunden Patienten mit kognitiven Störungen vor dem Hintergrund gut behandelbarer (z. B. depressiver) Erkrankungen vorstellig werden, die mittels der Lumbalpunktion zuverlässiger identifiziert werden können.

\section{Danksagung}

Wir danken Frau Stanislava Fockenberg (Fachärztin für Neurologie) und Herrn Diaa Rashid (Facharzt für Neurologie, Facharzt für Psychiatrie und Psychotherapie) für die Erstuntersuchung der Patienten, deren Zuweisung für die erweiterte Diagnostik im teilstationären Rahmen sowie für die Aufklärung und das Einholen der Einwilligung zur Studienteilnahme und Lumbalpunktion. Ebenso danken wir Frau Dr. rer. nat. Dipl.-Psych. Corinna Leonhardt und Frau M.Sc.-Psych. Meike Engel für die Durchführung der neuropsychologischen Untersuchungen und klinischen Ratings.

Interessenkonflikt

Die Autoren geben an, dass kein Interessenskonflikt besteht.

Literatur

[1] Wallesch CW, Förstl H. Klinische Diagnostik. In: Wallesch CW, Förstl H (Hrsg.) Demenzen. Stuttgart: Thieme; 2012:128-135

[2] Wiltfang J, Lewczuk P, Otto M. Biomarker bei Demenzen und anderen neurodegenerativen Erkrankungen. Aktuelle Entwicklungen. Nervenarzt 2016;87:1305-1309

[3] Magin P, Juratowitch L, Dunbabin J et al. Attitudes to Alzheimer's disease testing of Australian general practice patients: a cross-sectional questionnaire-based study. Int J Geriatr Psychiatry 2016;31:361-366

[4] Estrella M, Roberts A, Mäurer A et al. Akzeptanz der Demenzdiagnostik bei stationären, geriatrischen Patienten. Vergleich verschiedener 
Untersuchungsmethoden mit Fokus auf der FDG-PET-Untersuchung. Z Gerontol Geriatr 2017;50:52-58

[5] Robinson SM, Canavan M, O'Keeffe ST. Preferences of older people for early diagnosis and disclosure of Alzheimer's disease (AD) before and after considering potential risks and benefits. Arch Gerontol Geriatr 2014;59:607-612

[6] Boustani M, Perkins AJ, Fox C et al. Who refuses the diagnostic assessment for dementia in primary care? Int J Geriatr Psychiatry 2006;21:556-563

[7] Hurt CS, Burns A, Brown RG et al. Why don't older adults with subjective memory complaints seek help? Int J Geriatr Psychiatry 2012;27:394-400

[8] Cameron L, Leventhal EA, Leventhal H. Symptom representations and affect as determinants of care seeking in a community-dwelling, adult sample population. Health Psychol 1993;12:171-179

[9] Jessen F, Amariglio RE, van Boxtel $\mathrm{M}$ et al. A conceptual framework for research on subjective cognitive decline in preclinical Alzheimer's disease. Alzheimers Dement 2014;10:844-852

[10] Geerlings MI, Jonker C, Bouter et al. Association between memory complaints and incident Alzheimer's disease in elderly people with normal baseline cognition. Am J Psychiatry 1999;156:531-537

[11] Mitchel AJ. The clinical significance of subjective memory complaints in the diagnosis of mild cognitive impairment and dementia: a meta-analysis. Int J Geriatr Psychiatry 2008;23:1191-1202

[12] Jessen F, Wiese B, Bachmann C et al. Prediction of dementia by subjective memory impairment: effects of severity and temporal association with cognitive impairment. Arch Gen Psychiatry 2010;67:414-422

[13] DGPPN, DGN. S3-Leitlinie Demenzen.www.dgppn.de,www.dgn.de; Stand 2016

[14] CERAD-plus test battery. www.memoryclinic.ch; 2011

[15] Paajanen T, Hänninen T, Tunnard C et al. CERAD neuropsychological compound scores are accurate in detecting prodromal alzheimer's disease: a prospective AddNeuroMed study. J Alzheimers Dis 2014;39:679-690

[16] Morris J. Clinical Dementia Rating: A Reliable and Valid Diagnostic and Staging Measure for Dementia of the Alzheimer Type. Int Psychogeriatr 1997;9:173-176

[17] Herrmann C, Buss U, Snaith RP. HADS-D. Hospital Anxiety and Depression Scale - Deutsche Version. Bernd: Huber; 1995
[18] Sheik JI, Yesavage JA. Geriatric Depression Scale (GDS): Recent evidence and development of a shorter version. Clin Gerontol 1986;37:819-820

[19] Duits FH, Martinez-Lage P, Paquet $C$ et al. Performance and complications of lumbar puncture in memory clinics: Results of the multicenter lumbar puncture feasibility study. Alzheimers Dement 2016;12:154163

[20] Bohlken J, Köbe T, Dietz LM et al. Unterscheiden sich die Einstellungen von MCI-Patienten einer Gedächtnissprechstunde von denen einer Versorgerpraxis? Fortschr Neurol Psychiatr 2015;83:563-567

[21] Tang W, Kannaley K, Friedman DB et al. Concern about developing Alzheimer's disease or dementia and intention to be screened: An analysis of national survey data. Archives of Gerontology and Geriatrics 2017;71:43-49

[22] Vetter L, Keller M, Bruckner T et al. Adherence to the breast cancer surveillance program for women at risk for familial breast and ovarian cancer versus overscreening: a monocenter study in Germany. Breast Cancer Res Treat 2016;156:289-299

[23] Vogel A, Salem LC, Andersen BB et al. Differences in quantitative methods for measuring subjective cognitive decline - results from a prospective memory clinic study. Int Psychogeriatr 2016;28:1513-1520

[24] Kessler EM, Bowen CE, Baer M et al. Dementia worry: a psychological examination of an unexplored phenomenon. Eur J Ageing 2012;9:275284

[25] Boone KB. Fixed belief in cognitive dysfunction despite normal neuropsychological scores: neurocognitive hypochondriasis? Clin Neuropsychol 2009;23:1016-1036

[26] Orfei MD, Varsi AE, Blundo $C$ et al. Anosognosia in mild cognitive impairment and mild Alzheimer's disease: frequency and neuropsychological correlates. Am J Geriatr Psychiatry 2010;18:1133-1140

[27] Jessen $F$, Wolfsgruber $S$, Wiese $B$ et al. AD dementia risk in late $M C l$, in early $\mathrm{MCl}$, and in subjective memory impairment. Alzheimers Dement 2014; 10:76-83

[28] Luck T, Luppa M, Sieber J et al. Attitudes of the German general population toward early diagnosis of dementia - results of a representative telephone survey. PLoS One 2012;7:e50792 